\title{
A Novel Design of Voltage Controlled Oscillator by Using the Method of Negative Resistance
}

\author{
Ayoub Malki ${ }^{1}$, Larbi El Abdellaoui ${ }^{2}$, Jamal Zbitou ${ }^{3}$, A.Errkik ${ }^{4}$, A .Tajmouati ${ }^{5}$, Mohamed Latrach \\ 1,2,3,4,5LMEET Laboratory FST of Settat Hassan 1st University, Morocco \\ ${ }^{6}$ Microwave group ESEO Angers France
}

\begin{tabular}{l} 
Article Info \\
\hline Article history: \\
Received Mar 3, 2018 \\
Revised Jul 17, 2018 \\
Accepted Jul 30, 2018 \\
\hline Keyword: \\
Active device \\
Microstrip \\
Oscillator \\
Phase noise \\
Resonator \\
Voltage controlled oscillator
\end{tabular}

\section{INTRODUCTION}

The spectrum congestion of frequencies in the microwave field, the research for new applications in the telecommunication domain, and constraints of integration lead currently to the development of microwave systems. In regard to these systems, oscillators (simple or voltage controlled oscillator) are essential devices and their design presents many difficulties and challenges for laboratories and research centers. So such systems can't benefit a noticeable development unless their production price become reasonable; the difficulty of designing such circuits is therefore still spreading due to the seek for low costs. The solution is to design a monolithic or hybrid microwave integrated circuit realizing full integration of components in the same structure. This technology would allow the mass production of these structures in the

same series of operations [1]-[2].

\begin{abstract}
The objective of this paper is to develop a new design of a voltage controlled microwave oscillator by using the method of negative resistance in order to fabricate VCO with very good performance in terms of tuning rang, phase noise, output power and stability. The use of hybrid microwave integrated circuit technology's (HMIC) offers a lot of advantage for our structure concerning size, cost, productivity, and Q factor. This VCO is designed at [480MHz; $1.4 \mathrm{GHz}]$ frequency for applications in the phase locked loop (PLL) for signal tracking, FM demodulation, frequency modulation, mobile communication, etc. The different steps of studied voltage controlled oscillator's design are thoroughly described. Initially designed at a fixed frequency meanwhile the use of a varactor allow us to tune the frequency of the second design. It has been optimized especially regarding tuning bandwidth, power, phase noise, consumption and size of the whole circuit. The achieved results and proposed amendment are the product of theoretical study and predictive simulations with advanced design system microwave design software. A micro-strip VCO with low phase noise based on high gain ultra low noise RF transistor BFP 740 has been designed, fabricated, and characterized. The VCO delivers a sinusoidal signal at the frequency 480 $\mathrm{MHz}$ with tuning bandwidth $920 \mathrm{MHz}$, spectrum power of $12.62 \mathrm{dBm}$ into $50 \Omega$ load and phase noise of $-108 \mathrm{dBc} / \mathrm{Hz}$ at $100 \mathrm{~Hz}$ offset. Measurement results and simulation are in good agreement. Circuit is designed on FR4 substrate which includes integrated resonators and passive components.
\end{abstract}

Copyright $\odot 2018$ Institute of Advanced Engineering and Science. All rights reserved.

\section{Corresponding Author:}

Ayoub Malki,

University Hassan 1st,

University Complex Casablanca road, Km 3.5, B.P: 577 Settat, Morocco.

Email: ayoub.malki88@gmail.com

\footnotetext{
$$
\text { same series of operations [1]-[2]. }
$$
}


Voltage controlled oscillator are actually very important and indispensable module in the design of communication system employing in microwave technology. The objective's design is to get very stable output signal controlled in frequency with very low phase noise, power management (consumption, output power), pulling, matching impedance and the rejection of unwanted frequency bands. The role of the VCO is to provide approximately very perfect signal to the antenna to avoid the use of very high gain amplifier and the use of filters to bloc undesirable frequencies. Also the noise generates into the output signal of the VCO is susceptible strongly to degrade the sensitivity of the communication system. This noise called phase noise is distributed around the main frequency. In the other hand, in addition to the difficulty of VCO designs , the technological tendency, the requirements of the regulations and the speed of development of the telecommunications have increasingly forced to fabricate voltage controlled oscillator with very precise performances in term of size, consumption, phase noise, stability and output power. For that we present in this work, the study and complete design of a low phase noise voltage controlled oscillator, operating at [480MHz; $1.4 \mathrm{GHz}$ ] on epoxy FR4 with performances more better than founded in the literature.

We begin by presenting the oscillator theory including the fundamental oscillations conditions, the negative resistance structures and the hybrid integrated voltage controlled oscillator specifications. The choice of the topology is then argued. The last part of this work relates to the design process up to the fabrication and testing of our VCO. The importance of the layout realization step is widely developed. It allowed to proose an optimized and original architecture. The presentation and analysis of the obtained results with the published results in the literature positively end this work [3].

\section{FIXED FREQUENCY OSCILLATOR DESIGN}

In this paper, before to design voltage controlled oscillator with known tuning bandwidth we propose firstly to design an oscillator at fixed frequency to acquire the high performance of our oscillator. Secondly we replace the resonator of the fixed frequency oscillator by model of varactor based on diode to allow us to control the frequency of the voltage controlled oscillator.

An oscillator consist generally of four independent parts: matching network, active element (amplifier), resonator and bias network feedback element. During the procedure design, to improve the characteristic of the proposed model each component must be analyzed separately (choice of transistor, choice of positioning of tuning element, bias design and the architecture of the resonator). Finally the study of the interactive behaviors between the different components of oscillator is very important during the procedure design because this step allow us to get very precise performance of our structure [4].

The theory of oscillators can be firstly approached by the modeling of a counter-reacted system which is none other than the transmission approach shown in Figure 1(a). A non-linear amplifier with transfer function $\mathrm{G}$ is responsible for supplying the power to the load, it is then associated in the oscillator loop with a selective transfer function filter $\beta$ (f). It is therefore a positive feedback that reinjects part of the output signal at the input of the amplifier to build the oscillation [5]-[6].

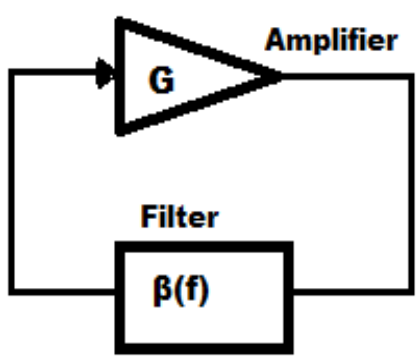

(a)

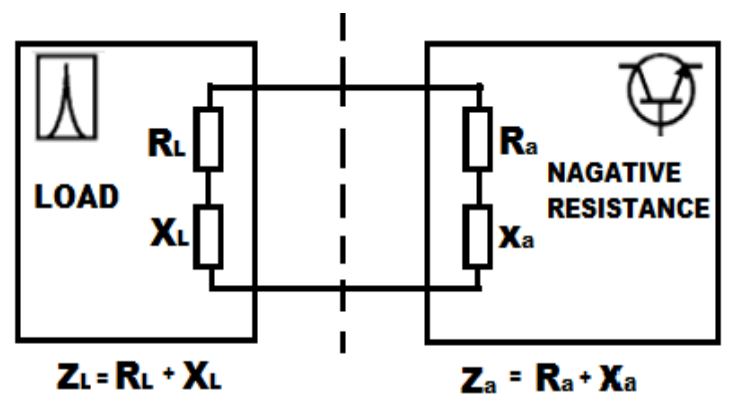

(b)

Figure 1. Oscillator as (a) feedback loop (b) negative resistance.

A sustained oscillation in steady state, requires that two conditions, known as the Barkhausen criterion, be simultaneously satisfied. Such a looped system reaches its stable oscillation state when the closed loop transfer function is exactly equal to 1 [7]. This implies that the total phase shift on a loop turn is zero. Mathematically the Barkhausen criterion can be written as follows: 


$$
\begin{aligned}
& \left\|\beta\left(f_{0}\right) * G\right\|=1 \\
& \Phi\left(\beta\left(f_{0}\right) * G\right)=0
\end{aligned}
$$

Although the model shown in Figure 1 (a) can be used to analyze and determine the necessary and sufficient conditions for oscillation, it is more judicious in the case of the design of VCO, to use the model shown in Figure 1 (b) whose analysis is performed using the concept of negative resistance. This concept explains that the tuned circuit, once it is supplied with voltage, will oscillate continuously if there is active device to compensate the resistance that absorb energy. The function of the amplifier therefore has the role of producing the negative resistance necessary to maintain the oscillation, ensuring a quantity of energy equal to that absorbed [8].

The energy in an oscillator is inevitably dissipated due to the non-ideality of the circuit elements. Therefore, energy must be used to maintain the oscillation. This can be achieved with the resulting negative resistance of transistor created by the transconductance $\mathrm{g}$ of transistor. For this study, an ultra-low noise SiGe transistor from Infineon, the BFP740, was chosen. Infineon BFP740 is a general purpose transistor that offers excellent performance at high frequency. The BFP740 is housed in a low cost low parasitic 3 lead F59. This transistor is recommended by Infineon to fabricate low-noise microwave oscillators or amplifiers and it delivers outstanding performance for a wide range of wireless application up to frequencies of $12 \mathrm{GHz}$. Its transition frequency is given at $150 \mathrm{GHz}$ and it has a ultra-low noise figure.

At the start of an oscillator the noise existing inside each electrical component must be excited to produce output signal. This excitation is the result of the negative energy created by the transistor. For the transistor deliver a sufficient amount of negative energy, it must be sufficiently unstable when the power source is connected to the system. This instability is translated by the module of return loss greater than the unit in the input port $\left(\left|\mathrm{S}_{11}\right|>\right)$ and the stability factor less than the unit $(|\mathrm{K}|<1)$. It's necessary that both ports of the amplifier are unstable because both sides of the amplifier serve a particular purpose in the built up of oscillation.

$$
K=\frac{1-\left|S_{11}\right|^{2}-\left|S_{22}\right|^{2}+\left|S_{11} S_{22}-S_{12} S_{21}\right|^{2}}{2\left|S_{21} S_{12}\right|}
$$

Based on the scattering parameters of BFP740 at common base given by the manufacturer Infineon at $1 \mathrm{GHz}$, we can conclude that the transistor operates at the stable area. In order to make it unstable we have added a feedback element. So by optimization of these feedback elements parameters and the adjustments of polarization parameters the transistor is becoming unstable.

The study of the active device stability in the common base configuration can be discussed using the ADS simulation specially the tool Stabfact to get the stability factor $\mathrm{k}$. This method consist to change the frequency around the frequency of oscillation sitting in the S-parameters component (start $=0.4 \mathrm{GHz}$ and $\mathrm{stop}=1.4 \mathrm{GHz}$ ). The resulting $\mathrm{k}$ factor versus frequency is shown in Table.1. The active device in a common base configuration with the feedback element are potentially unstable at [480MHz; $1.4 \mathrm{GHz}]$ [9].

Table 1. K Factor for Tte Active Device

\begin{tabular}{cc}
\hline Frequency & Sabfact:K \\
\hline $400 \mathrm{MHz}$ & -0.81 \\
$700 \mathrm{MHz}$ & -0.82 \\
$900 \mathrm{MHz}$ & -0.84 \\
$1.1 \mathrm{GHz}$ & -0.82 \\
$1.4 \mathrm{GHz}$ & -0.79 \\
\hline
\end{tabular}

The bias of the transistor is provided by a resistor bridge $\mathrm{R}_{1}, \mathrm{R}_{2}$ and a collector supply voltage of $5 \mathrm{v}$. A very detailed study is brought to see the different bias parameters because it plays a very important role to achieve the performances of our oscillator in term of power, efficient and phase noise. In this study we tried to have a stable transistor operation point and to minimize the power consummation because the characteristics of our transistor depend directly on its polarization [10].

An important issue in the design of a high performance oscillator is the design of a high quality resonator. As we have indicated, the quality factor has a significant impact on the performance of phase noise. It is therefore important to choose the high quality resonator in order to meet the best specifications. The chosen resonator is a micro strip resonator of length $\lambda / 2$. The circuit being in hybrid technology, the surface stress is much less than with an integrated circuit. The use of this type of resonator improves 
oscillator specifications better than LC resonator in terms of size and noise. The aim is to have inductance and capacitance where one can store energy without loss and at the same time the ability to integrate with a varactor. The quality factor of this micro strip resonator can be defined as follows:

$$
\begin{aligned}
& Q=\frac{w_{0}}{\Delta w} \\
& Q=2 \pi \frac{\text { Energie stokée }}{\text { Energie liberée par cycle }}
\end{aligned}
$$

At frequencies near of resonance frequency, the microstrip line can be replaced by an RLC series circuit whose values can be estimated using the following relationship:

$$
\begin{aligned}
& \mathrm{L}_{\mathrm{s}}=\frac{\mathrm{QR}}{\mathrm{w}_{0}} \\
& \mathrm{C}_{\mathrm{S}}=\frac{1}{\mathrm{QR}_{\mathrm{s}} \mathrm{w}_{0}}
\end{aligned}
$$

$R_{S}$ the series resistance determined by the actual part of the input impedance of the resonator at resonance, $\mathrm{W}_{0}$ is the pulsation at resonance, Ls inductance component of the resonator and $\mathrm{CS}_{\mathrm{S}}$ capacitance component of the resonator. To reduce the series resistance of the resonator we tried the maximum possible to reduce the width of micro strip lines. So a minimum characteristic impedance chosen is 25 .

The last step in this work is to design matching impedance. Impedance matching is a technique used in electricity to optimize the transfer of electrical power between a transmitter (source) and an electrical receiver (load) 1 and optimize the transmission of telecommunications signals.the theory of maximum power determines that the impedance of the load must be the conjugate complex of the impedance of the generator; Now the whole circuit including the active device with its polarization, resonator and matching impedance is as in Figure 2.

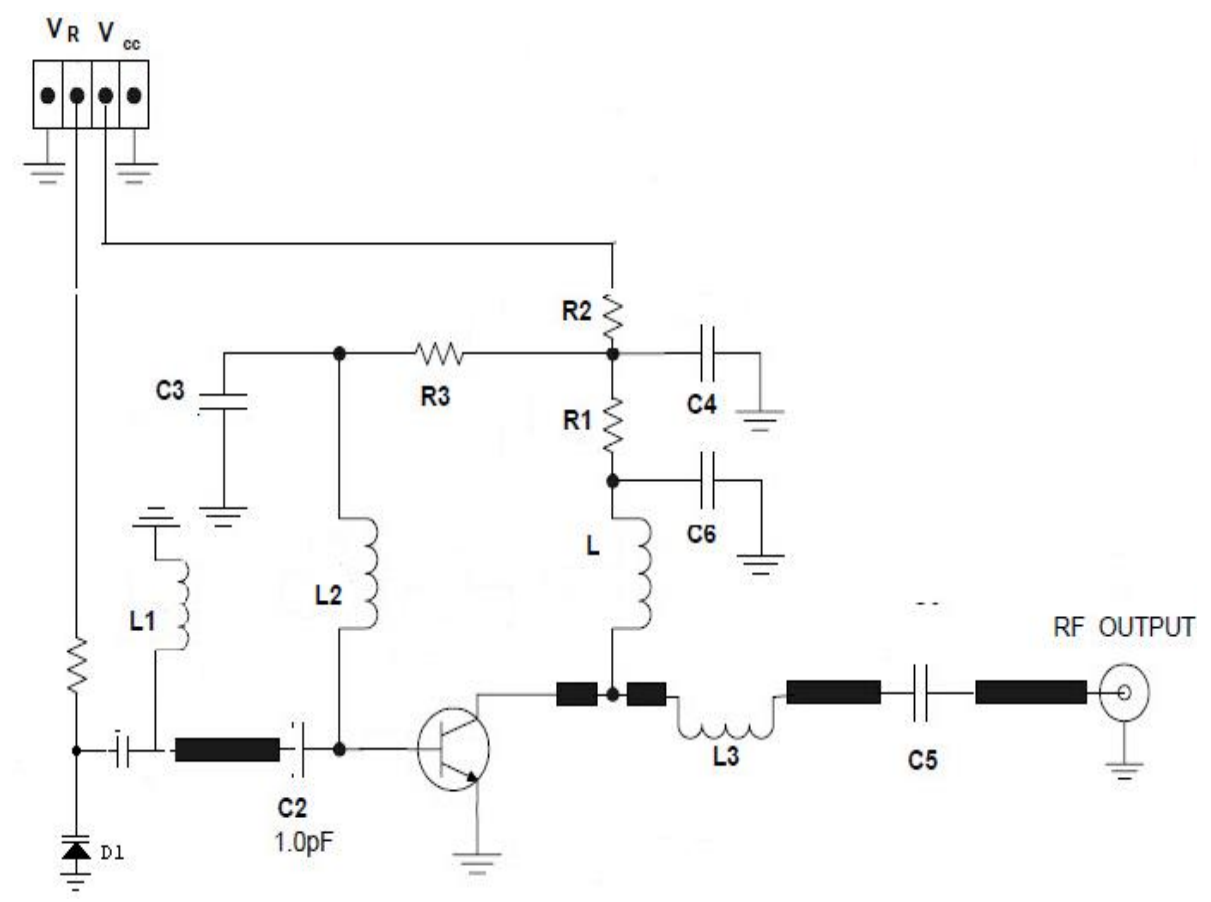

Figure 2. The schematic of the negative resistance microwave oscillator.

For the simulating of the obtained oscillator we can use the harmonic balance used for nonlinear structure which is frequency domain analysis tool. The frequency spectrum, the steady state and the phase noise results are shown successively in Figure 3 (a), Figure 3 (b) and Figure 3(c). 


\section{$\mathrm{m} 1$} harmindex $=1$ $\mathrm{dBm}($ HB. Vout1) $=14.909$

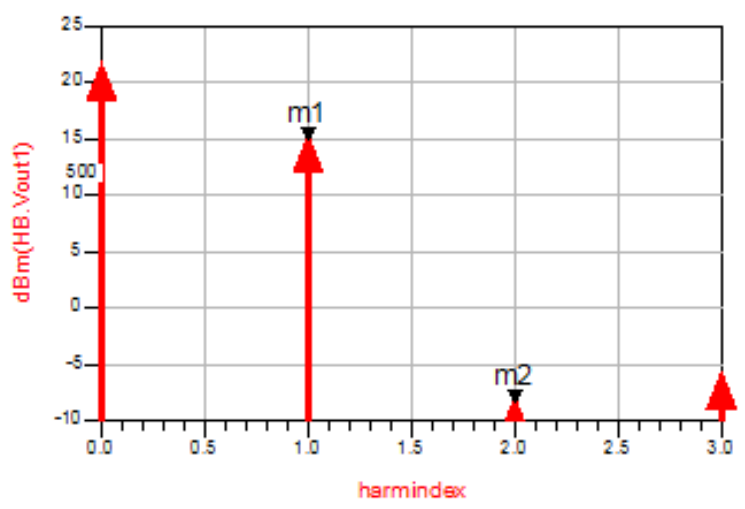

(a)

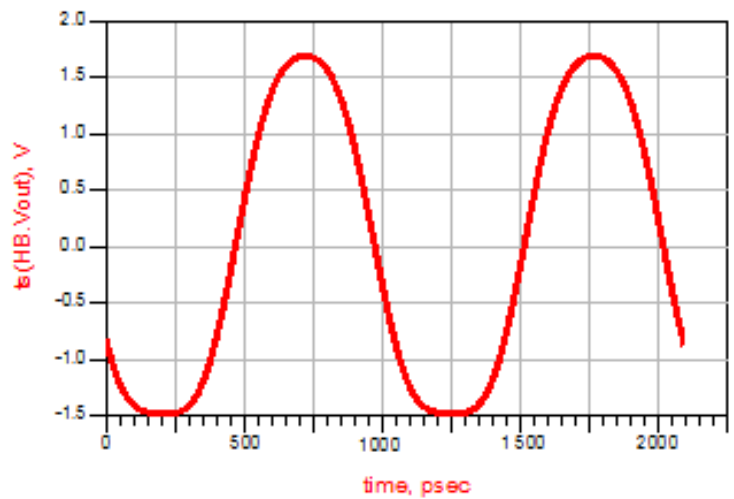

(b)

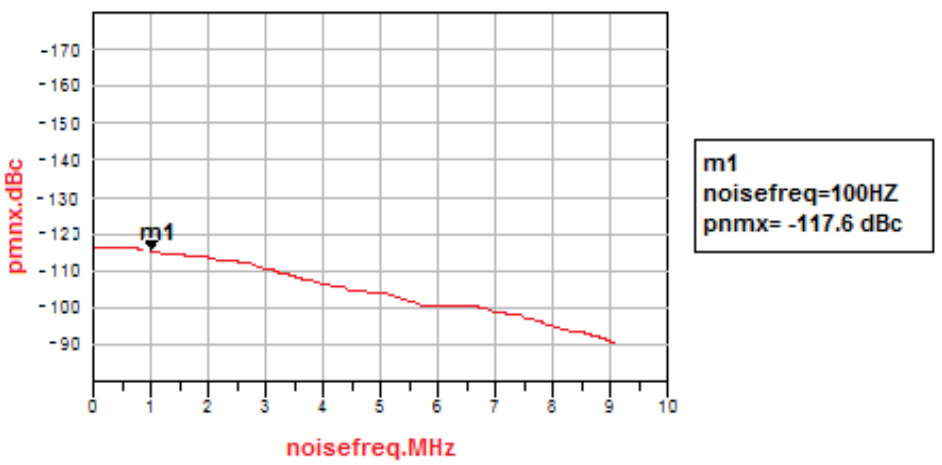

(c)

Figure 3. Simulated result of fixed oscillator in (a) time domaine (b) frequency domaine (c) phase noise.

It is seen from Figure 3 (a) that the waveform of the output signal is purely sinusoidal at $1 \mathrm{GHz}$. The output power at the fundamental frequency is $14.909 \mathrm{dBm}$ as shown in Figure 3 (b). We can also observe that the power of the harmonics is very negligible compared to the fundamental we can also observe that the power of the harmonics is very negligible compared to the fundamental one. Which further improves the distortion of the oscillator. In the author hand the phase noise prediction of the microwave oscillator is 117.6 $\mathrm{dBc} / \mathrm{Hz}$.

The proposed voltage controlled oscillator performances were compared to some existing works in the literature in terms of phase noise, output power and distortion, the comparison results conducts to the best results. Generally, in the literature, the best results are about $-110 \mathrm{dBc} / \mathrm{hz}$ from $100 \mathrm{~Hz}$ of the carrier in term of phase noise and $7 \mathrm{dBm}$ in term of output power. If we compare theses values with reached values in our work (phase noise: $-117.6 \mathrm{dBc}$ at $100 \mathrm{~Hz}$ from carrier; output power: $14.909 \mathrm{dBm}$ and the harmonic about -8.42 $\mathrm{dBm}$ ), we can conclude that the proposed VCO has excellent performances compared to the reported works.

\section{VOLTAGE CONTROLLED OSCILLATOR DESIGN}

In the following section the design of voltage controlled oscillator is given. This design example will use the same structure used in the fixed frequency version but we try to replace the resonator by varactor. The objective of this step is the frequency variation of the fixed frequency oscillator. This is allowed when the resonator parameters can be modified, namely its inductance and capacitance. There are many methods, each has their advantages and disadvantages. An integration of varicap based on diode is very adequate to our structure. The varactor diode, also called varicap diode, reverse polarized will have a variable capacitance between these two terminals.

In order to choose a suitable varactor for the design, we have to decide on the tuning bandwidth. This particular requirement is for a tuning bandwidth $45 \mathrm{MHz} / \mathrm{V}$ over the tuning range of 1 to $20 \mathrm{~V}$. To give 
us some margin to cover the external parasite effects we choose a bandwidth of $50 \mathrm{MHz} / \mathrm{V}$. The varactor should have a minimum of parasitism, so we opt for a SMT device. The simulation of the complete circuit with the varactor was run and re-run for each value of the voltage from $1 \mathrm{~V}$ to $20 \mathrm{~V}$. The results of variation voltage versus frequency and output power are given in Table 2.

Table 2. Tuning Frequency of VCO in Function of Voltage

\begin{tabular}{cc}
\hline Voltage & Frequency \\
\hline $0.5 \mathrm{~V}$ & $500 \mathrm{MHz}$ \\
$5 \mathrm{~V}$ & $700 \mathrm{MHz}$ \\
$10 \mathrm{~V}$ & $900 \mathrm{MHz}$ \\
$15 \mathrm{~V}$ & $1.1 \mathrm{GHz}$ \\
$20 \mathrm{~V}$ & $1.4 \mathrm{GHz}$ \\
\hline
\end{tabular}

\section{EXPERIMENTAL RESULTS AND DISCUSSION}

Good isolation between components as well as satisfactory decoupling between the DC ports and the RF lines are critical considerations for achieving an RF or microwave design. To avoid introducing asymmetry into the oscillation waveforms and to induce additional phase noise on the output signal, particular attention must be given to the symmetry of the complete layout. The voltage controlled oscillator was fabricated on the epoxy substrate with the dielectric constant of 4.4, the metal thickness of $0.035 \mathrm{~mm}$, and height is $1.60 \mathrm{~mm}$; as hybrid microwave integrated circuit structure. All the circuit components are in SMD (Surface Mount Device) package. Figure 4 illustrates the photograph of the completed fabricated voltage controlled oscillator. It occupies a surface of $28 * 30 \mathrm{~mm}^{2}$ integrating the pads of power supplies. Before the realization of the circuit, the electrical diagram extracted from the final layout, was simulated for the last time taking into account the given minimum and maximum values of the critical components (inductance, varactor, capacitances).

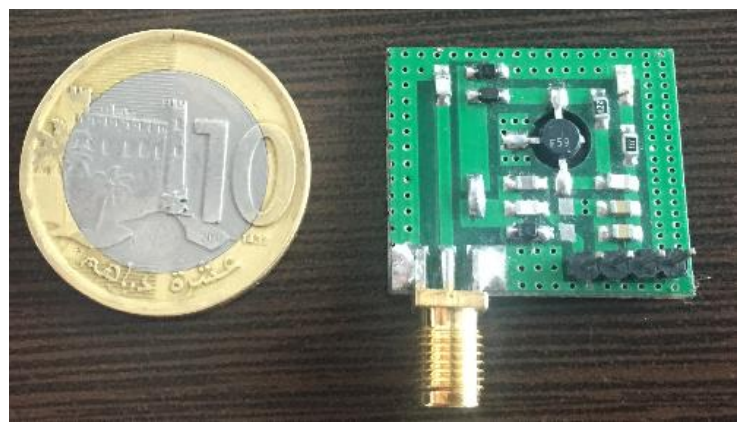

Figure 4. Photograph of the fabricated voltage controlled oscillator

The fabriquated voltage controlled oscillator is characterized succevelly in time domaine, frequency domain and phase noise using Tektronix DPO 7245 digital phosphor oscilloscope, Agilent E4440A PSA spectrum analyzer and Agilent 5505A. Figure 5 (b) shows that The VCO delivers $12.62 \mathrm{dbm}$ at $1 \mathrm{GHz}$, Figure 5(c) shows that phase noise levels of output signal is $-108 \mathrm{dBc} / \mathrm{Hz}$ at $100 \mathrm{~Hz}$ at an oscillation frequency of $1 \mathrm{GHz}$ and from Figure 5 (a) The output voltage has ideal sinusoidal shape with peak to peak voltage swing of $2.6 \mathrm{~V}$.

The measured and simulated parameters of our voltage controlled oscillator present approximately the same results in term of tuning bandwidth, frequency and output power and some offset in phase noise $(9 \mathrm{dBc})$. This offset between measured and simulated phase noise is normal due to the fabrication, approximations taken by ADS especially phase noise, the quality of components and the integration of the varicap.

If we compared the obtained results of this work (phase noise $-108 \mathrm{dBc} / \mathrm{hz}$, tuning bandwith 920 $\mathrm{MHz}$ ) with the published results of some scientific reports on microwave oscillator designs [11]-[17], we can conclude that this voltage controlled oscillator has good results in term of tuning bandwith, phase noise, good output power and frequency accuracy. These results were the product of deep study of each parameter of the voltage controlled oscillator and especially the interaction between these parametres. 


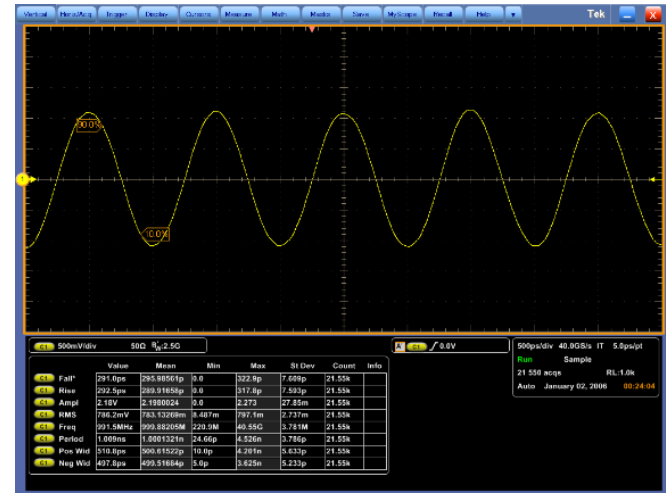

(a)

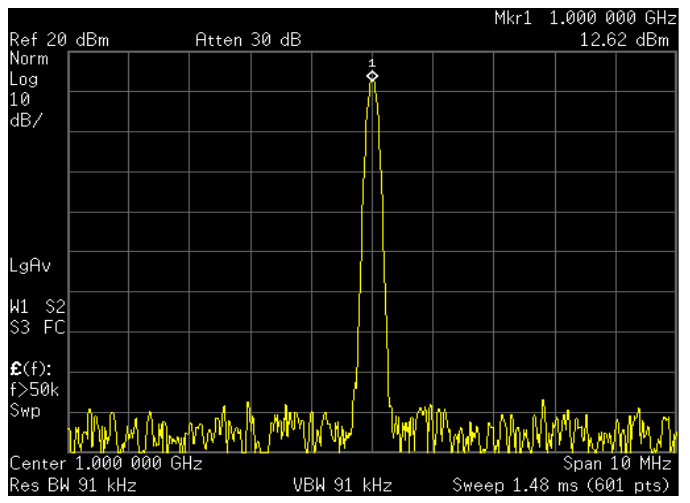

(b)

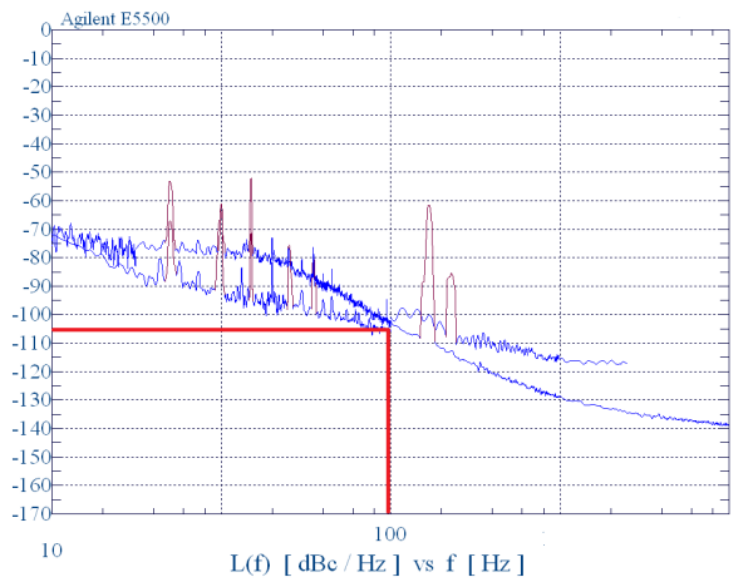

(c)

Figure 5. Measurement result of VCO in (a) time domaine (b) frequency domaine (c) phase noise.

\section{CONCLUSION}

As a conclusion, we can deduce that we have developed a novel structure negative resistance voltage controlled oscillator at $480 \mathrm{MHz}$ with tuning bandwith $920 \mathrm{MHz}$. The fabricated voltage controlled oscillator produces sinusoidal signal in frequency range $[480 \mathrm{MHz} ; 1.4 \mathrm{GHz}]$ with start frequency of $480 \mathrm{MHz}$, output power at $1 \mathrm{GHz}$ equal to $12.62 \mathrm{dBm}$, phase noise of the output signal around $-108 \mathrm{dBc} / \mathrm{Hz}$ at $100 \mathrm{~Hz}$. This work has been designed and adjusted by using a theoretical step and optimization tools applied on active device, Bias networks, matching impedance and resonator with its varicap,. The comparative study of this voltage controlled oscillator design with some summary of some scientific reports on voltage controlled microwave oscillator designs shows that we got better results.

\section{REFERENCES}

[1] M.M Elsherbini, M.F Elkordy, and A.M Gomaa, "Design and Simulation for UHF Oscillator using SAWR with Different Schematics, "Indonesian Journal of Electrical and Computer Science.Vol. 1, No. 2, pp. 294-299, February 2016.

[2] Kengo Kawasaki, Takayuki Tanaka, and Masayoshi Aikawa, "Ku band Second Harmonic N-Coupled Push-Push Oscillator Array using Microstrip Resonator, " 2010 Proceedings of the IEEE MTT-S on Microwave Symposium Digest,Anaheim,2010,pp. 1182-1185

[3] T. Ohira, "Rigorous Q-factor formulation for one- and two-port passive linear networks from an oscillator noise spectrum viewpoint," 2005 IEEE Transactions on Circuits and Systems II: Express Briefs, vol. 52, no. 12, pp. 846850, 2017.

[4] D.M Pozar, " Microwave engineering, " John Wiley .1989.

[5] Bhan u Shrestha, N. Kim, "Low phase noise microwave oscillator using meander spurline resonator for X band application," Indian journal of engineering materials sciences, Vol. 18, pp. 381-384, October 2011. 
[6] Bhavana benakaprasad, Salah sharabi, K. Elgaid, "RF and Microwave oscillator design using p-HEMT transistor," International journal of scientific and research publication, Vol. 04, No 8, pp. 1-7, August 2014.

[7] A. Hajimiri, T.H.Lee, "The design of low noise oscillator," Norwell .1999.

[8] K. Chang, I. Bahl, V. Nair. Editors, "RF and microwave circuit and component design for wireless systems," Wiley 2002 .

[9] Achmad Munir, Endon Bharata, "Self oscillating mixer with dielectric resonator for low noise block application," TELKOMNIKA telecommunication computing electronics and control, Vol. 09,No 2, pp. 351-356, August 2011.

[10] Khairun Nisa' Minhad, Zainab Kazemi, Mamun Bin IbneReaz, Jubayer Jalil, Noorfazila Kamal, "Design of a Current Starved Ring Oscillator Based VCO for Phase-Locked Loop," Indonesian Journal of Electrical and Engineering, Vol.12,No 9, pp. 6667-6672, September 2014.

[11] R.A. Pucel, R. Bera, D. Masse, "Experiments on integrated gallium-arsenide FET oscillators at X band," Electronics letters.vol. 11, no. 10, pp. 219-220, 1975.

[12] O. Ishihara, T. Sawano, M. Nakatani, "Highly stabilized GaAs FET oscillator using a dielectric resonator feedback circuit in 9-14 GHz," IEEE transaction on microwave theory and techniques. vol. 28, no. 08, pp. 817-824, 1980.

[13] S. Chen, S. Tadayon, T. Ho , K. Pande, P. Ria, J. Adair, M. Ghahrenani, "U-band MMIC HBT DRO. IEEE microwave and guided wave letters," vol. 04, no. 02, pp. 50-52, 1994.

[14] H.Jacobsson, S. Gevorgian, M. Mokhtari , C. Hedenas , B. Hansson , T. Lewin , H. Berg, W. Rabe , A. Schuppen, "Low phase noise low power IC VCO for 5-8 GHz wirless application, " IEEE MTT-S integration microwave symposium. vol. 48, no. 12, pp. 723-726, 2000.

[15] Hun-Wah Fan, K. -K. M. Cheng," New method in characterizing the nonlinear current model of MESFET using single-tone excitation, "IEEE MTT-S International on Microwave Symposium Digest. pp. 449-452, 2000.

[16] D.Kuylenstiena, S.lai, M.Bao, H. Zirath, "Design of low phase noise oscillators and wideband Ward VCOs in InGap HBT technology," IEEE Transactions on Microwave Theory and Techniques. vol. 60, no. 11, pp. 34203430, 2012.

[17] Madureira, N.Deltimple, E.Kerherve, "Design and measurement of class EF2 power oscillator," Electronics Letters. vol. 51, no. 10, pp. 05-14, 2015.

\section{BIOGRAPHIES OF AUTHORS}

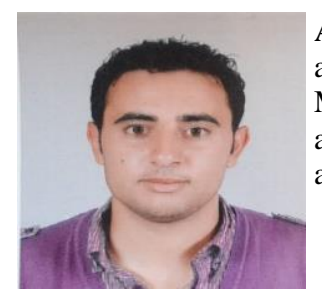

Ayoub Malki was born in Settat, Morocco, In january 1988. He received the master degree in aeronautic and telecommunications from Air royal scool, University of kadi Aayad Marrakech, Morocco. He is currently working toward the Ph.D. degree in physics and engineering sciences at Faculty of Sciences and Technologies, Settat, Morocco. His research interests include the analysis and design of hybrid, monolithic active and passive microwave electronic circuits.

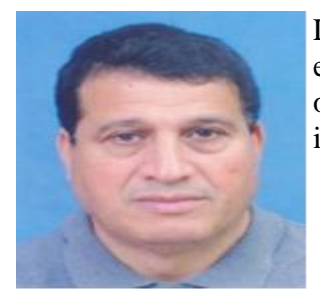

Larbi El Abdellaoui was born in Tiflet, Morocco, in 1961. He received the Ph.D. degree in electronics from the University of Metz, in 1994, France. He is currently an associate Professor of Electronics in Faculty of sciences and techniques, University Hassan 1st, Settat, Morocco. He is involved in the design of hybrid, monolithic active and passive microwave electronic circuits.

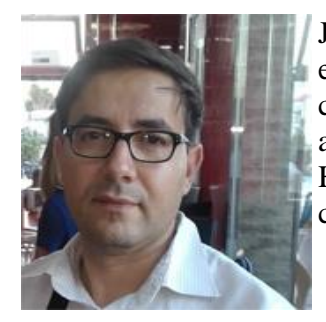

Jamal Zbitou was born in Fes, Morocco, in June 1976. He received the Ph.D. degree in electronics from Polytech of Nantes, the University of Nantes, Nantes, France, in 2005. He is currently an associate Professor of Electronics in FST, University Hassan 1st, Settat, Morocco and the head of Computing Networks and telecommunication in LMEET Laboratory in FSTS. $\mathrm{He}$ is involved in the design of hybrid, monolithic active and passive microwave electronic circuits.

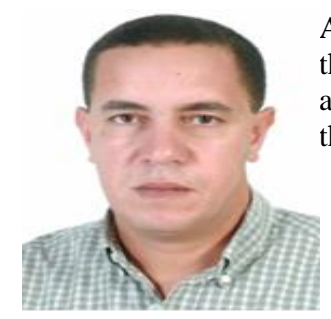

Ahmed Errkik was born in July 1960 in Morocco. He received the Ph.D. degree in physics from the University of Technology Compie'gne (UTC), Compie gne, France. He is currently an associate Professor of physics in FST University Hassan 1st, Settat, Morocco. He is involved in the design of hybrid, monolithic active and passive microwave electronic circuits. 


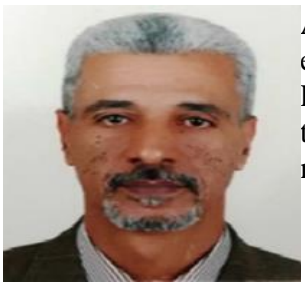

Abdelali Tajmouati was born in, Morocco, in 1962. He received the Ph.D. degree in science engineering from the University of Perpignan, France, in 1992. He is currently an associate Professor of Electronics, thermal transfer and thermodynamic in Faculty of sciences and techniques University Hassan 1st, Settat, Morocco. He is involved in the design of hybrid, monolithic active and passive microwave electronic circuits.

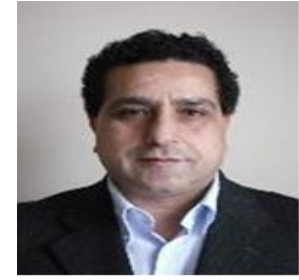

Mohamed Latrach (IEEE member) received the Ph.D. degree in electronics from the University of Limoges, Limoges, France, in 1990. He is currently Professor of microwave engineering with the Ecole Supérieure d'Electronique de l'Ouest (ESEO), Angers, France, where he is head of the Radio-Frequency \& Microwave research group. His research interests include: design of hybrid \& monolithic active and passive microwave circuits, metamaterials, LH materials, antennas, rectennas and their applications in wireless communications, and wireless power transmission (WPT). 